\title{
Kernos
}

Revue internationale et pluridisciplinaire de religion grecque antique

7| 1994

Varia

\section{Du bon usage de l'oracle de Delphes}

\section{Jules Labarbe}

URL : http://journals.openedition.org/kernos/1109

DOI : 10.4000/kernos.1109

ISSN : 2034-7871

\section{Éditeur}

Centre international d'étude de la religion grecque antique

\section{Édition imprimée}

Date de publication : 1 janvier 1994

ISSN : 0776-3824

\section{Référence électronique}

Jules Labarbe, « Du bon usage de l'oracle de Delphes », Kernos [En ligne], 7| 1994, mis en ligne le 20 avril 2011, consulté le 06 mai 2019. URL : http://journals.openedition.org/kernos/1109 ; DOI : 10.4000/kernos. 1109 
Kernos , 7 (1994), p. 219-230.

\section{DU BON USAGE DE L'ORACLE DE DELPHES}

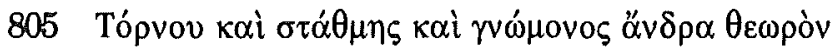

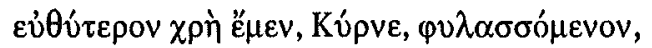

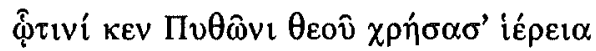

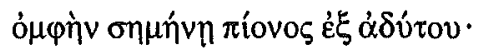

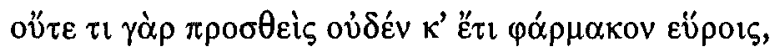

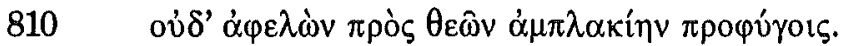

Ainsi s'exprime Théognis (v. 805-810) dans une des multiples pièces de vers élégiaques qu'il a destinées à son ami Cyrnos. Ce n'est pas la plus claire de toutes. Les modernes se sont ordinairement contentés d'en donner une explication assez sommaire où logique et cohérence laissent à désirer. Elle mérite, je pense, un nouvel examen.

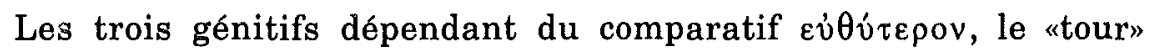

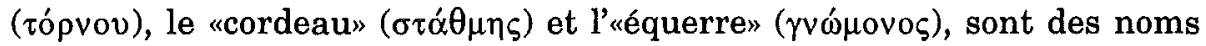
d'instruments qui évoquent en commun une idée fondamentale de rectitude, de rigueur: il ne saurait y avoir d'hésitation là-dessus.

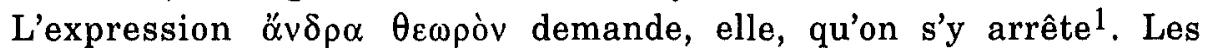
dictionnaires renvoient au poème de Théognis en accordant au nom apposé le sens de "spectateur" ${ }^{2}$, qui est attesté dans la meilleure langue ${ }^{3}$, mais qui, dans le cas présent, ne s'adapte pas au contexte,

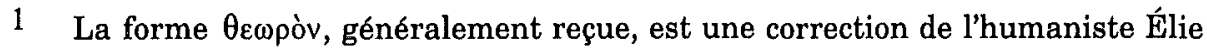
Vinet (1509-1587), qui écrivit une traduction latine de Théognis. Les mss ont $\theta \varepsilon \omega \rho \hat{v}$, leçon qu'a défendue Th.W. ALLEN (Adversaria IV, in RPh, 3e série, 11 [1937], p. 286) : ce serait un génitif partitif. La langue usuelle couple $\alpha \dot{\alpha} p$ avec un substantif en apposition au même cas : cf. R. KÜHNER (- B. GERTH), Ausführl. Gramm. der griech. Spr., Satzlehre, 3e éd., I (Hanovre, 1898), p. 271272. Le génitif partitif est toutefois possible : Allen invoque notamment $\tau \hat{\omega} \mathrm{v}$

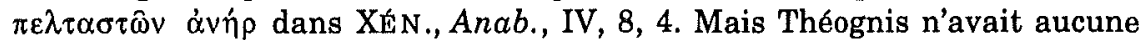
raison d'isoler un membre dans un groupe forcément visé en entier par ses observations : «un" théore doit être rigoureux, non pas «un d'entre les théores».

2 LIDDELl-ScotT-JoNes, Gr.-Engl. Lex. ${ }^{9}$, s.v. $\theta \varepsilon \omega \rho$ ó $\zeta$, III; BAILly (-SÉCHAN-

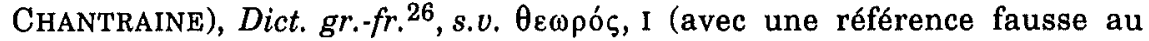
v. 803).

3 Voir Eschyle, Choéph., 246; Prom., 118; fr. 289 Nauck $^{2}$; Platon, Rép., V, 467c; Timée, 57d; A RSTT., Pol. , VII, 1336b 36... 
celui-ci ne mentionnant rien qui soit expressément offert aux regards. Dans les inscriptions comme chez les auteurs, les $\theta \varepsilon \omega \rho$ oi sont d'ordinaire des envoyés officiels; ils représentent leurs cités respectives aux fêtes panhelléniques ou dans l'accomplissement de tâches religieuses à l'étranger. Cette dernière activité peut prendre la forme d'une visite à un sanctuaire délivrant des oracles ${ }^{4}$. Nous voilà apparemment sur le bon chemin puisque c'est de la Pythie de Delphes qu'il va être question. Le théore dont parle Théognis est-il membre d'une commission de l'espèce, d'une $\theta \varepsilon \omega \rho i ́ \alpha$ chargée d'interroger Apollon et de rapporter la réponse divine à la cité qui l'a mandatée ? Les

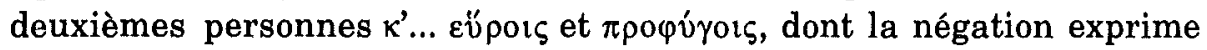
visiblement les fâcheuses conséquences d'une absence d'exactitude, ne pourraient avoir trait à une cité mandante que si elles étaient susceptibles d'une valeur indéterminée ("on ne trouverait pas", "on n'éviterait pas" - le "on" désignant les citoyens). Déjà peu plausible en

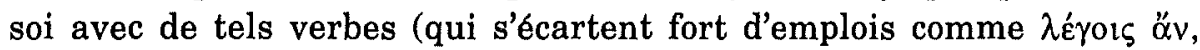

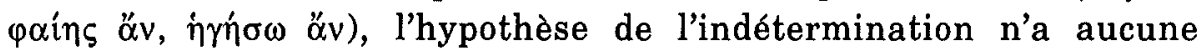
vraisemblance après la phrase où figure le vocatif Kúpve. C'est en Cyrnos lui-même que Théognis voit le théore exposé à des difficultés notables. S'il n'en allait pas de la sorte, les deuxièmes personnes demeureraient injustifiées. En effet, proposé à l'attention de Cyrnos sous un aspect strictement objectif et sans être identifié avec lui, l'ỏvì $\rho$ $\theta \varepsilon \omega \rho$ òs aurait été sujet de verbes à la $\mathrm{tr}$ o i si èm $\mathrm{e}$ personne. Semblablement, - les exigences métriques mises à part, - les verbes se trouveraient à la $\mathrm{p} \mathrm{r}$ e $\mathrm{m}$ i è r e personne si, comme la supposition en a été émise, Théognis remplissait ou avait rempli les fonctions de théore et désirait, à partir de sa propre expérience, souligner une obligation inhérente à leur exercice 5 .

4 Voir sur les diverses acceptions, outre Souda, $\Theta 225$ : Cl.P. BILL, Notes on the Greek $\Theta \varepsilon \omega \rho o ́ \varsigma$ and $\Theta \varepsilon \omega \rho i \alpha$, in TAPhA, 32 (1901), p. 196-197; G. BusoLT, Griech. Staatsk., 3e éd., I (Munich, 1920), p. 508; D. WACHSMUTH, art. Theoria, -oi, in Der kl. Pauly, V, 24 (1974), col. 730-731. Et aussi P. ChANTRAINe, Dict. étym. de la langue gr., Paris, 1968, p. 433-434 [avec des remarques critiques sur l'explication habituelle par $* \theta \varepsilon \bar{\alpha}-(F)$ opós, $* \theta \varepsilon \eta-(F)$ opó $\varsigma>\theta \varepsilon(\varepsilon) \omega \rho o ́ s$, combinant $\theta \varepsilon$ é $\alpha$ spectacle» et $-(F)$ opos «qui observe»; sur la tentative de $\mathrm{H}$. Koller au

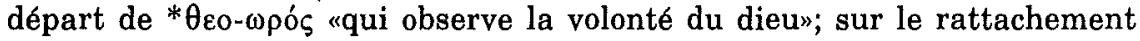

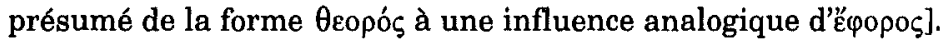

5 C.M. Bowra (Two poems of Theognis (805-810 and 543-546), in Philologus, 103 [1959], p. 157-166) veut trouver dans les v. 543-546, apparentés à 805-810

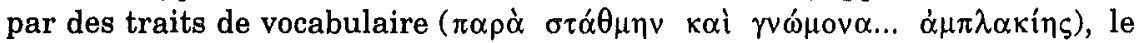
signe que Théognis exerçait une magistrature à Mégare et, dès lors, une 
Peut-on croire que Cyrnos, conformément à une idée assez répandue, ait été désigné ou sur le point d'être désigné par sa cité, par Mégare, pour aller consulter l'oracle de Delphes à titre officiel ${ }^{6}$ ? Qu'on l'imagine seul dans cette charge ou membre d'une commission, on se heurte à une difficulté majeure. Elle tient à la nature des suites

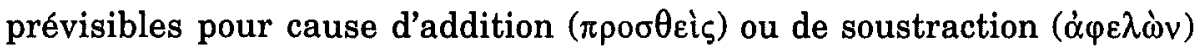
intempestive. Ces suites ne seraient pas indiquées pour la cité présumée mandante, mais seulement pour son délégué Cyrnos, simple intermédiaire. Or c'est elle, et non lui, qui, en bonne logique, aurait eu besoin d'un $\varphi \alpha ́ \rho \mu \alpha \kappa o v$ bénéfique et ne le trouverait pas dans le message déformé ${ }^{7}$. L'obstacle n'est pas levé si l'on adopte l'interprétation dramatisante selon laquelle Cyrnos, devenu criminel en raison de son manque de rectitude, n'aurait pas de remède, son acte accompli, pas de recours contre le châtiment, et s'attirerait forcément, de la part des dieux, un verdict de culpabilité ${ }^{8}$. Le terme $\varphi \alpha ́ \rho \mu \alpha \kappa o v$ est inapproprié comme désignation d'un moyen permettant de ne pas être puni et l'adverbe ë $\tau$ se comprendrait mal, qui implique la disparition d'une latitude précédemment accordée ${ }^{9}$.

confirma tion de sa possible activité comme $\theta$ zopò (forme dorienne) de la cité. Mais en 543-546, Théognis parle à la première personne, ce qu'il ne fait pas dans l'autre poème. L'hypothèse de Bowra était esquissée dans J. C ARRIÈRE, Théognis de Mégare. Etude sur le recueil élégiaque attribué à ce poète, Paris, 1948, p. 180, n. 5. Plus anciennement dans Fr. CAUER, Studien zu Theognis, in Philologus, 51 (1892), p. 305. Et déjà dans Th. BERGK, Poetae lyrici graeci, 4e éd., II (Leipzig, 1882), p. 189, par le détour de corrections, à vrai dire aussi vaines qu'audacieuses : $\mu \varepsilon \quad \chi \rho \hat{\eta} . . . \varphi v \lambda \alpha \sigma \sigma \sigma \varepsilon ́ \mu \varepsilon v \alpha$.

6 E. BuchHolz (- R. PePPMÜLleR), Anthologie aus den Lyrikern der Griechen, 6e éd., I (Leipzig, 1925), p. 105; B.A. VAN GRONINGEN, Théognis. Le premier iivre, Amsterdam, 1966, p. 309; J. Carrière, Théognis. Poèmes élégiaques, nouv. éd., Paris, 1975 (C.U.F.), p. 177.

7 Le décalage doit n'avoir pas gêné E.BuchHolz (- R. PEPPMÜLLER), qui, reconnaissant expressément en Cyrnos un délégué officiel, commentent (l.l.) [c'est moi qui souligne] : «fälschest du..., so findest $d u$ keine Rettung, wie $d u$ sie suchst...".

8 Ce que croit B.A. VAN GroningEN, op. l., p. 310-311.

9 Les éditeurs impriment communément $\kappa^{\prime}$ ětı au v. 809, soit comme leçon du ms. le plus ancien, A (s. X), soit (ainsi fait M.L. West) en interprétant ce qui, en réalité, serait sa scriptio continua $\kappa \varepsilon \tau$. La variante largement attestée $\kappa \varepsilon ́ \tau \imath$ doit

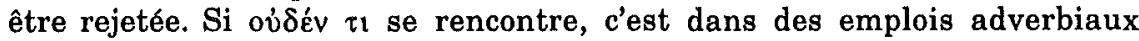
(comme HDT., V, 65, 1; XÉn., Anab., VII, 6, 26; Hell., III, 2, 14; cf. SoPH., Ajax,

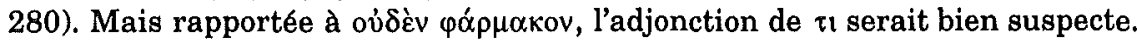


Pour appuyer l'idée que les vers 809-810 concernent d'imparables sanctions qu'encourrait le théore indélicat, maint érudit a invoqué une glose de la Souda ${ }^{10}$ :

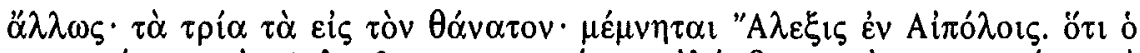

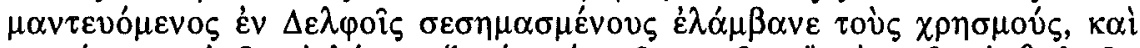

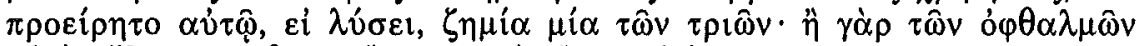

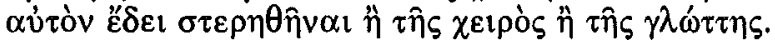

Pris à partir de ö $\tau 1$, le texte peut se traduire comme suit :

(...) le consultant, à Delphes, recevait les oracles cachetés et on lui avait préalablement annoncé, s'il en rompait les sceaux, un châtiment parmi ces trois-ci : il devait être privé ou bien de ses yeux, ou bien de sa main, ou bien de sa langue.

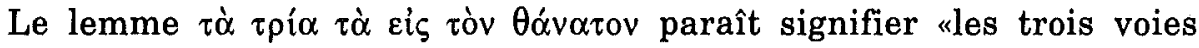
vers la mort». En réalité, on a affaire ici à la s e c on de partie, introduite par $\alpha \lambda \lambda \omega \varsigma$ ("Autre note"), d'une glose assez étendue relative à

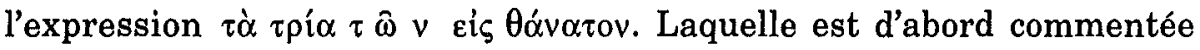
en ces termes:

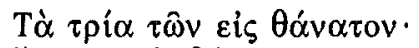

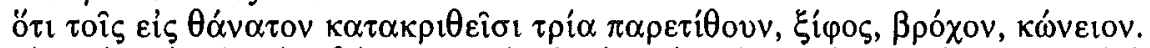

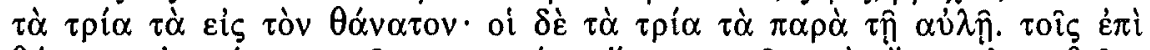

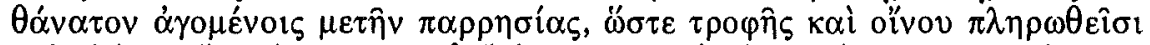

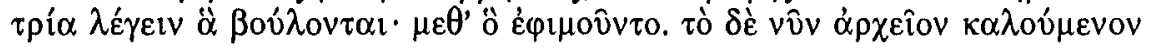

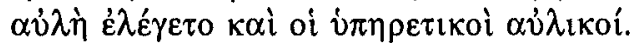

"Les trois choses" des hommes <conduits> à la mort.

Aux hommes condamnés à mort on offrait trois possibilités : le glaive, le nœud coulant, la ciguë - les trois voies vers la mort. D'aucuns mettent «les trois choses" en rapport avec la Cour <des exécutions> : aux hommes que l'on conduisait à la mort était accordée une liberté de parole, le droit, une fois gorgés de nourriture et de vin, de dire trois choses qu'ils voulaient $<$ dire $>-$ après quoi on les bâillonnait. (C'est ce qui maintenant s'appelle Archeion que l'on dénommait «Cour <des exécutions》" et "gens de la Cour» ceux qui étaient à son service.)

La Souda commence donc par reproduire deux interprétations d'une expression à caractère proverbial qui évoquerait des aspects de la mise à mort : soit certains modes d'exécution, soit une faveur accordée in extremis aux condamnés. Pénétrons-nous de ce début de la notice pour

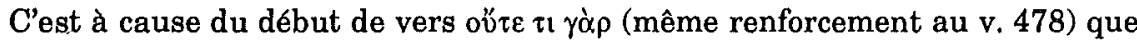

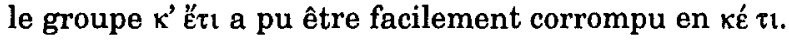

10 Souda, T 154. 
bien comprendre la portée de ce qui vient s'ajouter après $\alpha \lambda \lambda \lambda \omega \varsigma$. Les

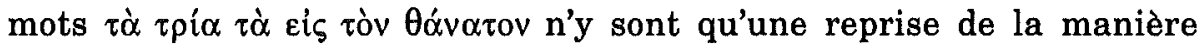
qui a servi à caractériser le groupe constitué par le glaive, le nœud coulant et la ciguë ${ }^{11}$. Pour le sens, et même syntaxiquement ${ }^{12}$, ils

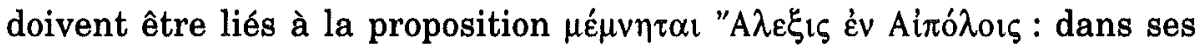
Chevriers, dont il ne subsiste aucun autre fragment, Alexis, poète de la comédie moyenne, faisait apparemment mention des trois sortes de mort violente. La proposition ö $\tau$ ó $\mu \alpha v \tau \varepsilon v o ́ \mu \varepsilon v o \varsigma \kappa \tau \lambda$., d'ailleurs précédée d'une ponctuation dans l'édition de la Souda, ne dépend pas de $\mu \varepsilon ́ \mu \nu \eta \tau \alpha \mathrm{l}$ et ne rend pas un renseignement tiré d'Alexis. Elle ne vise plus la nature ni les à-côtés de l'exécution des condamnés, mais extrapole, pour ainsi dire, en rattachant à la formule glosée l'indication, avec le banal ö $\tau$ introductif d'une scholie, de cruels $\tau p i \alpha$ qui, pourtant, ne sont pas

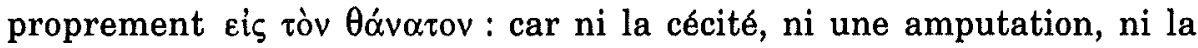
mutité n'empêchent un homme de survivre. Le parémiographe Zénobe, dans un témoignage parallèle à celui de la Souda ${ }^{13}$, enlève son anonymat à la proposition en cause : il intercale après Ainóioıs, suivi d'un

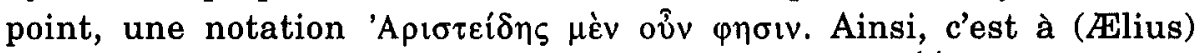
Aristide, dont l'intérêt pour Delphes est démontrable ${ }^{14}$, qu'il convient d'attribuer la spécification du traitement promis au consultant infidèle: non point la mort, mais d'être rendu soit aveugle, soit manchot, soit muet - punitions correspondant à trois sortes précises de

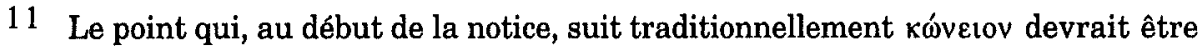
remplacé par une virgule ou par un tiret (comme dans ma traduction), de manière à mieux faire apparaître le rôle d'apposition assignable, en cet endroit,

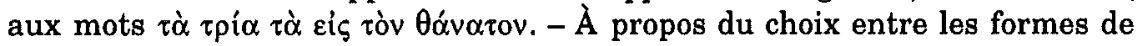
mort, il existe ailleurs une expression $\tau \hat{\omega} v$ $\tau$ pเôv $\ddot{v} v$ qui évoquait, semble-t-il, une mesure prise par Théramène sous le gouvernement des Trente : voir ARISTOPHANE, fr. 563 Kassel-Austin (= 549 Kock); HÉsychios, T 1754; PHOTIOS, Lex. , II, p. 235 Naber; Souda, T 871.

12 À condition de supprimer le point en haut marqué avant $\mu \varepsilon ́ \mu v \eta \tau \alpha$.

13 ZÉnOBE, 6, 11, dans Corpus Paroem. Gr., I, p. 164 Leutsch-Schneidewin, ainsi que dans M.E. Miller, Mél. de litt. gr., Paris, 1868, p. 355-356. C'est la version de Zénobe qui a été retenue pour AlExis, fr. 8, dans J.M. EDMONDS, The Fragments of Attic Comedy, II (Londres, 1959), p. 376-379. Au lieu du nom "A $\lambda \varepsilon \xi \xi \zeta$, le C.P.Gr. offre une variante 'A $\lambda \hat{\xi} \xi \alpha \nu \delta \rho o \zeta ;$ elle doit être d'autant moins prise en considération que, dans le ms. édité par Miller, il y a bel et bien "A $\lambda \varepsilon \xi_{1 \varsigma}$, comme dans la glose de la Souda.

14 Voir surtout ÆLIUS A RISTIDE, Rhét., 1, 34-41 (I, 2, p. 155-156 Behr). Et cf. P. Amandry, La mantique apollinienne à Delphes, Paris, 1950, p. 261, renvoyant à ses remarques sur des passages d'Aristide relatifs à l'oracle. 
violation d'un texte secret (par lecture individuelle, par remise à autrui, par communication de vive voix). L'étude de la documentation littéraire et épigraphique a montré que, si les réponses oraculaires, à Delphes, pouvaient être données oralement, elles tendaient tout de même ensuite à prendre d'ordinaire, sauf lors de consultations privées, une forme écrite ${ }^{15}$. Ce qui a embarrassé, dans l'information fournie par Alius Aristide, c'est qu'elle semble en contradiction avec l'usage, bien attesté, qui amenait les théores à connaître le texte qu'ils devaient transmettre consigné, éventuellement en l'ayant noté de leur main, à être familiarisés avec sa teneur, avant que vînt le jour où les sceaux pourraient être rompus, date que Zénobe souligne, dans sa rédaction propre, en

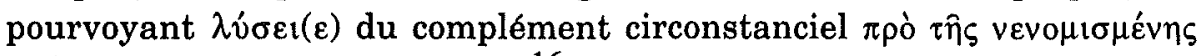
$\grave{\eta} \mu \varepsilon ́ p \alpha \varsigma$, "avant le jour normal» ${ }^{16}$. Ællius Aristide n'est pas nécessairement indigne de foi. Peut-être parlait-il d'une cité particulière - mais pour quelle époque? - qui, dérogeant à la coutume, avait de redoutables exigences et se montrait d'une rare sévérité envers la délégation qu'elle envoyait à Delphes.

De toute façon, le théore de Théognis n'est pas exposé, lui, à un quelconque des trois châtiments. L'oracle ne lui est pas rendu par écrit,

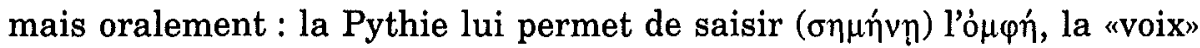
d'Apollon ${ }^{17}$. Rien ne suggère une transcription subséquente. Le

15 P. AMANDRY, op. l., p. 150 et n. 3.

16 Cf. C.M. BOWRA, l.l. (n. 5), p. 159-160.

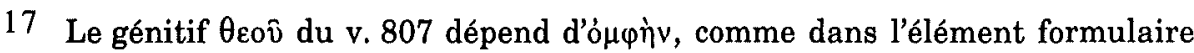
homérique de $\gamma 215$ et $\pi 96$, mais, étant donné sa place, rien ne s'oppose à ce

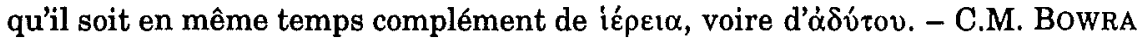
(l.l., p. 158) rappelle le mot fameux d'HeRACLITE, fr. 93 Diels $^{6}$ (-Kranz) : «le

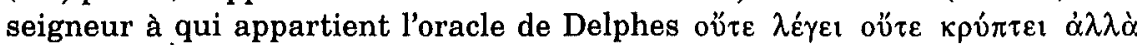

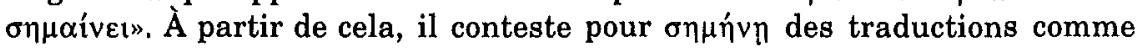

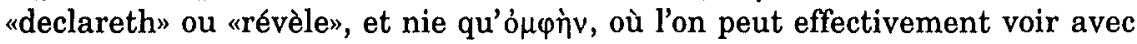
lui un complément d'objet direct de $\chi \rho \eta \dot{\sigma} \sigma \alpha \sigma^{\prime}$, dépende - disons : en même temps - du verbe au subjonctif. Sa position n'est en tout cas pas conforme au fait que le nom ó $\mu \varphi \hat{n}$, s'il signifie la "voix", peut désigner aussi, occasionnellement, les mots qu'elle prononce, le message qu'elle apporte : voir les vers homériques $\gamma 215$ et $\pi 96$ mentionnés plus haut, où on lit é $\pi$ $\sigma \pi$ ó $\mu \varepsilon v o r$

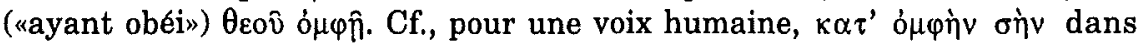

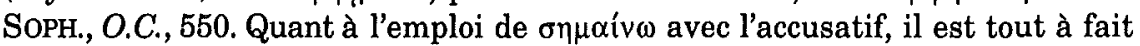
banal au sens de "signaler, indiquer, annoncer, communiquer", p. ex. dans HDT., I, 5, 3; 43, 3; III, 14, 8, etc. On notera surtout HDT., VII, 18, 3, où l'invitation qu'Artabane fait à Xerxès de communiquer aux Perses ce qu'il a lui-même vécu

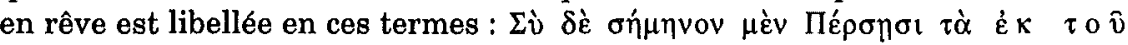
$\theta \varepsilon \circ \hat{v} \pi \varepsilon \mu \pi \hat{\alpha} \mu \varepsilon v \alpha$. 
participe $\varphi \nu \lambda \alpha \sigma \sigma o ́ \mu \varepsilon v o v$ a tout l'air d'interdire qu'il puisse, en l'occurrence, y en avoir une. Il est généralement perçu comme exprimant la circonspection, le zèle attentif indispensable au théore ${ }^{18}$. On lui assigne ainsi le rôle d'un qualificatif attribut, mais son absence de coordination

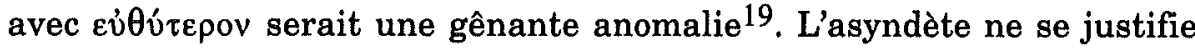
ici que si le participe équivaut à une proposition subordonnée circonstancielle. Plutôt qu'une insistance sur le devoir de rigueur que formule

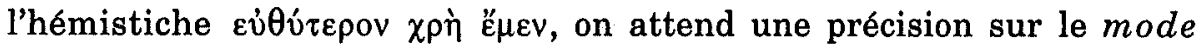
d'action pour lequel le devoir est imposé. Dès lors, il y a tout lieu de croire que, comme plus d'une fois dans la langue archaïque, et même

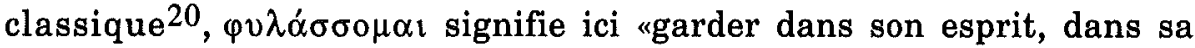

18 T. HudSON-WILLIAMs, The Elegies of Theognis and other Elegies included in the Theognidean Sylloge, Londres, 1910, p. 226 : «being on his guard»; J.M. EDMONDS, Elegy and Iambus, I (Londres, 1931), p. 325 : «must... be diligent»; J. Carrière, Théognis. Poèmes élégiaques, Paris, 1948 (C.U.F.), p. 64 : «il doit s'efforcer à plus d'exactitude»; nouv. éd., Paris, 1975, p. 102 : «il doit s'astreindre à plus de rigueur»; A. GARZYA, Teognide. Elegie, Florence, 1958, p. 101 : "occorre... che... attento vada"; Fr. R. ADRADOS, Líricos Griegos elegiacos y yambógrafos arcaicos (siglos VII-V A.C.), II (Barcelone, 1959), p. 220 : "Con más exactitud... y lleno de precauciones dobe»; B.A. VAN GRONINGEN, op. $l$. (n. 6), p. 310 : «il faut qu'il se tienne sur ses gardes».

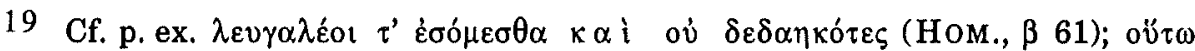

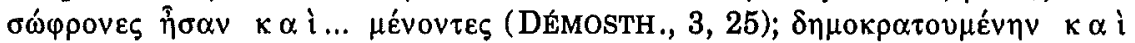

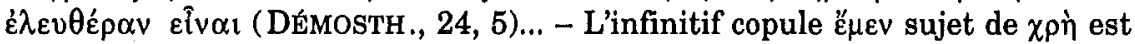
une conjecture admise par une moitié des éditeurs du XXe siècle, l'autre moitié

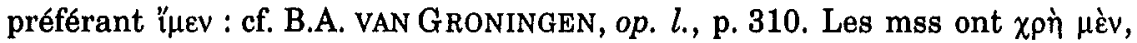
sans accents dans le plus ancien, A (s. X), selon l'apparat critique de M.L. WEST (Iambi et elegi graeci ante Alexandrum cantati, 2e éd., I [Oxford, 1989], p. 212). Il suffirait en principe, pour avoir un texte convenable, de considérer la deuxième syllabe comme liée à la première par un phénomène d'aphérèse $(\chi \rho \grave{n}$ ' $\mu \varepsilon v)$. Mais vu l'extrême rareté de ce phénomène dans le lyrisme ancien (cf. R. KÜHNER [- Fr. Blass], Ausführl. Gramm. der griech. Spr., Elementarund Formenlehre, 3e éd., I [Hanovre, 1890], p. 241), mieux vaut la munir d'un

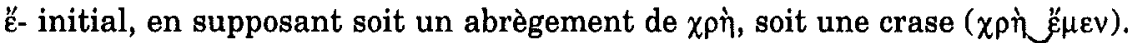
Certes, la prononciation iotacisante de $\chi \rho \hat{~}$ expliquerait tout aussi bien une disparition de la lettre initiale d'i $\mu \varepsilon v$, mais ce verbe est nettement moins bien approprié qu'é $\mu \varepsilon v$, l'idée de "aller, marcher droit" s'accordant mal avec les termes de comparaison du v. 805 et n'étant en rien justifiée par un rappro-

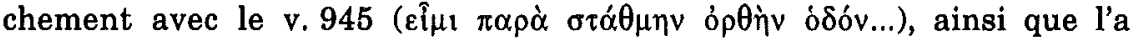
souligné T. Hudson-Williams (l.l.). De toute manière, même avec ‘uev, le participe $\varphi v \lambda \alpha \sigma \sigma o ́ \mu \varepsilon v o v$, au sens où on l'entend d'ordinaire, apparaît irréguliè-

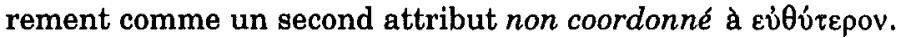

20 Cette signification, garantie par le complément circonstanciel dans HÉs., Trav.,

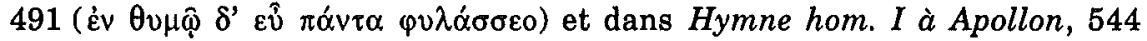


mémoire», $\varphi \nu \lambda \alpha \sigma \sigma o ́ \mu \varepsilon v o v$ correspondant sensiblement à une subordonnée ó $\alpha \nu$ $\varphi \nu \lambda \alpha ́ \sigma \sigma \eta \tau \alpha 1$. Le complément d'objet direct n'est pas exprimé, mais il se supplée sans peine à partir du contexte, où ce qu'il faut retenir avec un scrupule extrême ne tardera pas à être explicité, dans le distique suivant (v. 807-808), par ó $\mu \varphi \eta ́ v$, terme précis ressortant nettement au début du "pentamètre».

Cyrnos délégué effectif ou potentiel de sa cité, de Mégare, c'est, on l'a vu plus haut, une hypothèse insatisfaisante. Je voudrais en avancer une

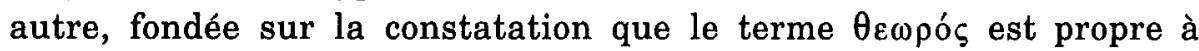
désigner aussi l'homme qui va consulter un oracle à titre strictement personnel. Un passage de Sophocle témoigne clairement de pareil emploi. Dans l'EEdipe Roi, en effet, répondant à une question d'CEdipe sur les circonstances dans lesquelles Laïos a été assassiné, Créon précise, à propos de ce dernier (v. 114-115) :

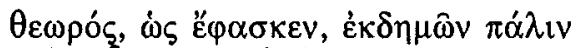

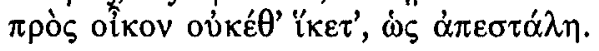

Théore consultant à l'étranger selon ses dires, il ne revint plus au logis, une fois qu'il s'en fut allé.

Aussi malavisés que dans la traduction de leur référence à Théognis, les dictionnaires invoquent le premier de ces vers, en même temps que le v. 413 de l'Edipe à Colone, pour étayer l'acception d'uenvoy sent to consult an oracle», de "député envoyé par les États grecs... pour consulter un oracle». Dans l'EEdipe à Colone, c'est bien de délégués

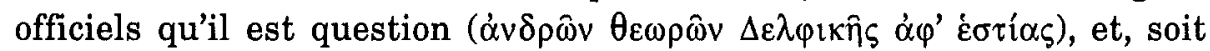
dit en passant, ils rapportent une réponse orale (v. $415: \dot{\omega} \varsigma \varphi \alpha \sigma i v$ oi

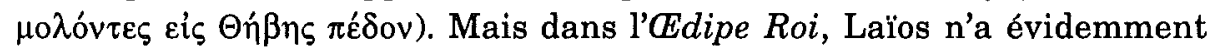
pas joué le même rôle qu'eux. Le verbe $\alpha \pi \varepsilon \sigma \tau \alpha \dot{\alpha} \lambda \eta$ ne veut pas dire qu'il ait été "envoyé", mais, conformément à un usage tragique du passif

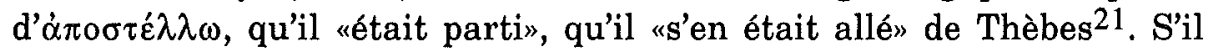

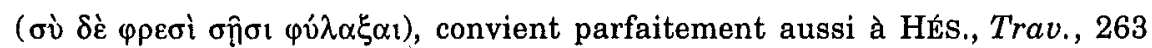

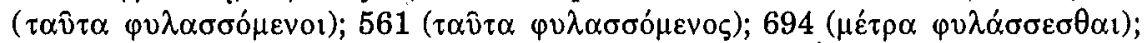

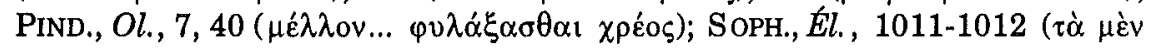

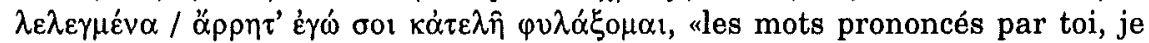
les garderai dans ma mémoire [en les traitant] comme non-dits et sans conséquence").

21 Sur ce point du moins, on peut se référer à LIDDELL-SCOTT-JONES, Gr.-Engl. Lex. ${ }^{9}$, s.v. $\dot{\alpha} \pi \sigma \sigma \tau \varepsilon \dot{\lambda} \lambda \omega$, I : «Pass. go away, depart, S. OT 115». Sont cités en outre : Eur., Suppl., 582; Hél., 660; Phén., 319; Iph. en Taur., 1409. Voir schol.

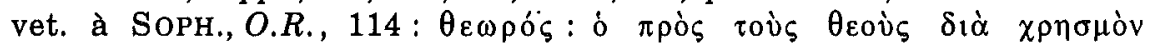


voulait se rendre à Delphes, - dont les environs ont été donnés comme le théâtre de sa mort violente, - c'était de son propre mouvement. Il détenait un pouvoir royal : qui donc aurait eu le droit de lui confier une mission? Les déclarations plutôt discrètes dont Créon fait état ( $\omega \varsigma$ $\ddot{\varepsilon} \varphi \alpha \sigma \kappa \varepsilon v)$ prouvent qu'il n'était pas mandaté, que personne n'avait organisé son voyage et qu'il n'avait pas fourni de détails sur le motif de sa consultation 22 .

Un cas assez semblable au sien - dans un contexte moins bien défini cependant - est fourni par l'Hippolyte d'Euripide, où Thésée, roi d'Athènes, quand il rentre inopinément à Trézène, son lieu d'exil provisoire (v. 34-37), s'étonne de n'être pas accueilli plus joyeusement malgré sa qualité de «théore» (v. 792 : $\dot{\omega} \zeta \theta \varepsilon \omega$ pòv) et, apprenant la mort de Phèdre, rejette la couronne de feuillage qu'il avait ceinte, puisqu'il

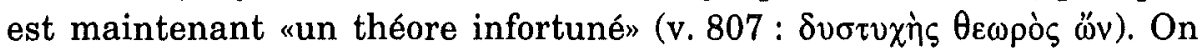
ne sait pas d'où il revient, mais c'est vraisemblablement de Delphes, où, à en croire les traditions suivies par Plutarque, il aurait fait plusieurs visites, en recevant de la Pythie plusieurs réponses ${ }^{23}$; les $\pi \lambda \varepsilon \kappa \tau \dot{\alpha}$ $v^{\prime} \lambda \lambda \alpha$ qu'il porte à son entrée en scène ne sont d'ailleurs pas sans analogie avec le laurier dont Créon apparaît couronné, dans l'Edipe Roi, au retour d'une consultation delphique qu'il a jugée encourageante ${ }^{24}$.

Si Théognis s'adresse à Cyrnos en le sachant ou en l'imaginant sur le point d'aller à Delphes en simple particulier, à titre personnel, pour

$\dot{\alpha} \pi \varepsilon \rho \chi$ ó $\mu \varepsilon \vee$ ○ . Ou Manuel Moschopoulos, dans O. Longo, Scholia byzantina in Sophoclis Oedipum Tyrannum, Padoue, 1971, p. $13: \dot{\alpha} \pi \varepsilon \sigma \tau \alpha \dot{\alpha} \lambda \eta$

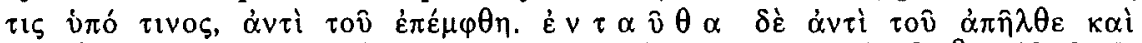
$\dot{\alpha} \pi \varepsilon \delta \eta_{\mu} \eta \eta \varepsilon \varepsilon$. Vont dans le même sens : L. CAMPBELL, Sophocles ${ }^{2}$, I (Oxford, 1879), p. 148 («After he had once taken his departure»); R.C. JEBB, Sophocles, I (Cambridge, 1887), p. 27 («after he had once set forth); P. MASQUERAY, Sophocle, 3e éd., I (Paris, 1940), p. 145 ("après qu'il fut parti»); P. MAzoN, Sophocle, II (Paris, 1958), p. 76 («du jour qu'il en fut parti»); J. BoLLACK, L'Edipe Roi de Sophocle, I (Lille, 1990), p. 189 («ainsi qu'il s'en était allé»).

22 Le voyage de Laïos à Delphes, EURIPIDE (Phén., 35-37) le dit motivé par le désir de savoir si l'enfant jadis exposé n'était plus en vie. Mais Sophocle a pu avoir une autre idée : peut-être Laïos s'inquiétait-il des "troubles of the state", comme dit L. CAMPBELL (l.l. ) - en particulier, des ravages causés par la Sphinx (L. CAMPBell - Ev. AвBOTT, Sophocles, rééd., II [Oxford, 1899], p. 16).

23 Plut., Thésée, 18, 3; 24, 4-5; 26, 5.

24 SopH., O.R. , 63. Mais Créon, lui, est allé à Delphes en mission, comme messager et pour le compte du souverain (v. 69-72). 
ses propres affaires ${ }^{25}$, le poème devient plus intelligible. Moyennant l'approfondissement que réclame encore son dernier distique.

Le pó $p \mu \alpha \kappa o v$ dont Cyrnos serait privé de manière définitive par une addition malencontreuse qu'il introduirait dans le texte à garder en mémoire n'est pas, je l'ai souligné plus haut, le moyen d'éviter un affreux châtiment. Ce qu'il faut y voir, c'est tout simplement le remède indiqué par Apollon Pythien pour sortir de la situation difficile ou confuse qui aura fait l'objet de la consultation. Corrélativement, l'ò $\mu \pi \lambda \alpha \kappa$ ín inévitable en cas de soustraction ne désigne ni une infraction grave ni un état de culpabilité du point de vue des dieux ${ }^{26}$. Théognis, qui affectionne le mot dirait-on, l'emploie certes au sens de "faute", voire de "crime» 27 , mais il lui donne aussi celui de "désarroi", d'uégarement».

Ainsi, au v. 404, à propos de l'homme qui, espérant un profit, mani-

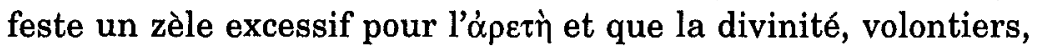

$$
\begin{aligned}
& \text { (...) mène à un grand é g a re me } \mathrm{nt}
\end{aligned}
$$

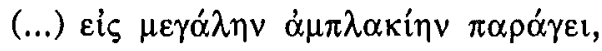

après avoir troublé son sens des valeurs, au point qu'il croit «bon ce qui est mauvais, et mauvais ce qui est profitable",

- au v. 630 , où l'on apprend que fleur de l'âge et jeunesse rendent léger l'esprit de l'homme

et de beaucoup de gens poussent l'âme à l'é g a r e m e n t

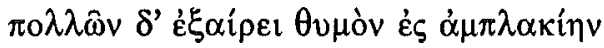

25 Comme le fait, p. ex., Xénophon avant de partir pour l'Asie : XéN., Anab., III, 1, 5-7. - Peut-être n'est-il pas inutile de noter que la présence d'óvíp à côté de

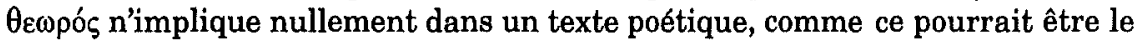
cas dans un texte de prose, la mise en relief d'un titre officiel ou d'une fonction reconnue : cf. R. KÜHNER (- B. GERTH), l.l. (n. 1).

26 Significations retenues par E. BUCHHOLZ (- R. PEPPMÜLLER), l.l. (n. 6) («... wirst du der Verschuldung... nicht entgehen") et par divers érudits mentionnés supra, n. 18 : T. HUDSON-WILLIAMS, l.l. («Avoid the charge of sin..."); J.M. EDMONDS, l.l. («escape offence»); J. CARRIÈRE, $l l . l l$. («comment ne serait-on

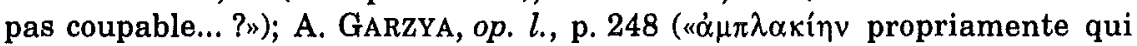
'accusa di colpa'»); Fr. R. AdrAdos, op. l., p. 221 («acusado de culpa»). Pour B.A. VAN GRONINGEN, voir supra, p. 215 et n. 8 .

27 Théognis, 204 (crime effectivement commis), 386 (faute, infraction), 546 (manquement dans l'exercice d'une magistrature : cf. supra, n. 5). 
- au v. 632, où celui dont la tête ne domine pas le cœur est dit se trouver

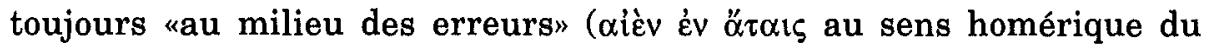
terme)

\section{(...) outre qu'il gît en de grands dé s a r r o is

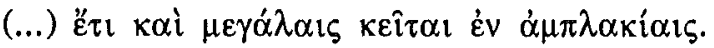

L'acception en cause est celle qui convient au v. 810. L'interrogation de l'oracle - habituelle en deux questions constituant une alternative tend à obtenir un avis sur la conduite à tenir dans telle ou telle circonstance. Tronquer la réponse reçue, faute d'une bonne mémorisation, c'est en perdre le bénéfice et se condamner à une incertitude où le monde divin $(\theta \varepsilon \hat{\omega} v)$ - et pas seulement le dieu de Delphes $(\theta \varepsilon o \hat{v})$ - verra le risque inéluctable de dangereux écarts.

Les conséquences de l'action d'ajouter ( $\pi \rho \circ \theta \theta \varepsilon i \varsigma)$ et de l'action d'ôter (ỏ $\varphi \varepsilon \lambda \dot{\omega} v$ ) sont présentées sous des formes distinctes (d'une part, perte de l'ultime remède possible, d'autre part, entrée dans un état d'égarement), mais il va de soi qu'elles se rejoignent et se confondent. Leur dissociation est purement rhétorique et l'inversion des causes ne changerait rien à l'idée de base, qui est la dénonciation des dangers inhérents à toute modification quantitative d'un texte oraculaire mal gravé dans l'esprit ${ }^{28}$.

La traduction suivante essaie de tenir compte des résultats acquis au fil de l'analyse :

Plus que le tour, le cordeau et l'équerre un consultant doit être rigoureux, Cyrnos, quand il garde en mémoire,

lui pour qui, à Pythô, la prêtresse du dieu, en rendant un oracle, aura fait s'exprimer sa voix hors du somptueux saint des saints.

C'est qu'avec un rien d'ajouté, tu ne pourrais plus trouver nul remède, non plus qu'avec un rien d'ôté, éviter ce qui est, aux yeux des dieux, l'égarement.

28 «Quantitative» si l'on entend de manière stricte les verbes $\pi \rho \circ$ o

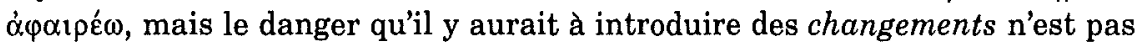
exclu pour autant. En effet, le complément d'objet direct $\tau$ du v. 809, à reprendre au v. 810 , ne se limite pas forcément à la désignation d'un mot ou d'un groupe de mots : il peut concerner une idée en plus ou en moins qui serait due à une altération lexicale, morphologique ou syntactique. - Au v. 810 , ov้ $\tau^{\prime}$ des recentiores est une lectio facilior, au point de vue syntactique, pour ov́ $\delta$ ' (Ao), qui marque tout à fait normalement une insistance sur la formulation

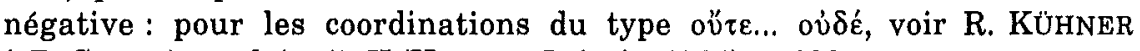
(- B. GERTH), op. l. (n. 1), II (Hanovre-Leipzig, 1904), p. 290, g. 
Dans les considérations de ses quatre premiers vers, le poème a un caractère général, mais son distique final montre que l'intention de Théognis était d'expliquer ce que devait être pour son ami, en tant que simple particulier, une consultation delphique efficace. Il ne faut donc pas voir ici un témoignage sur l'institution appelée $\theta \varepsilon \omega \rho i ́ \alpha$ dans les États grecs, ni sur leurs précautions en matière oraculaire, sanctions à l'appui. On retiendra, en revanche, de l'avertissement à Cyrnos qu'il constitue, selon toute apparence, une exaltation de la mémoire, de son importance, de la précision qu'elle exige - ce qui ne saurait surprendre à cette époque où la pratique de l'écriture ne l'emportait pas encore sur l'oralité 29 .

Rue des Orchidées, 92

Jules LABARBE

B - 4030 LIÈGE

29 Cf. ma communication Survie de l'oralité dans la Grèce archaïque, in Phoinikeia Grammata. Lirc et écrire en Méditerranée, Liège-Namur, 1991, p. 499-531, notamment p. 519-522. 